\title{
14-3-3 Protects against stress-induced apoptosis
}

\author{
C Clapp ${ }^{1,4}$, L Portt $^{1,4}$, C Khoury ${ }^{2}$, S Sheibani ${ }^{1}$, G Norman ${ }^{1}$, P Ebner ${ }^{2}$, R Eid ${ }^{1}$, H Vali ${ }^{3}$, CA Mandato ${ }^{3}$, F Madeo ${ }^{2}$ and MT Greenwood ${ }^{\star, 1}$
}

Expression of human Bax, a cardinal regulator of mitochondrial membrane permeabilization, causes death in yeast. We screened a human cDNA library for suppressors of Bax-mediated yeast death and identified human 14-3-3 $\beta / \alpha$, a protein whose paralogs have numerous chaperone-like functions. Here, we show that, yeast cells expressing human 14-3-3// $\alpha$ are able to complement deletion of the endogenous yeast 14-3-3 and confer resistance to a variety of different stresses including cadmium and cycloheximide. The expression of 14-3-3 $\beta / \alpha$ also conferred resistance to death induced by the target of rapamycin inhibitor rapamycin and by starvation for the amino acid leucine, conditions that induce autophagy. Cell death in response to these autophagic stimuli was also observed in the macroautophagic-deficient atg1 $\Delta$ and atg7 4 mutants. Furthermore, 14-3-3 $\beta / \alpha$ retained its ability to protect against the autophagic stimuli in these autophagic-deficient mutants arguing against so called 'autophagic death'. In line, analysis of cell death markers including the accumulation of reactive oxygen species, membrane integrity and cell surface exposure of phosphatidylserine indicated that 14-3-3 $\beta / \alpha$ serves as a specific inhibitor of apoptosis. Finally, we demonstrate functional conservation of these phenotypes using the yeast homolog of 14-3-3: Bmh1. In sum, cell death in response to multiple stresses can be counteracted by 14-3-3 proteins.

Cell Death and Disease (2012) 3, e348; doi:10.1038/cddis.2012.90; published online 12 July 2012

Subject Category: Experimental Medicine

Programmed cell death (PCD) is a critically important process that has a multitude of purposes in metazoans. It is involved in sculpting the final body that results after development and it serves to remove cells that may be a threat to the organism be they damaged or be they infected with foreign invaders., PCD is also of clinical importance as it is disrupted in a number of diseases. ${ }^{3-5}$ Inhibitors of the process would be beneficial in numerous pathophysiological situations where cell death is increased such as after ischemic/reperfusion events that are commonly seen in the heart. ${ }^{4}$ Similarly, the ability to selectively increase PCD in tumors represents another major impetus for much of the research that focuses on understanding the mechanisms regulating $\mathrm{PCD}{ }^{3}$

Our understanding of PCD has increased greatly over the last few years with the identification and characterization of novel negative regulators of different forms of PCD, including necroptosis. ${ }^{2,5-7}$ The identification of enzymes like deoxyuridne triphosphate (dUTPase), that utilize dUTP as a substrate, as a prosurvival sequence has served to focus attention on the fact that reactive oxygen species (ROS), calcium and ceramide are not the only second messengers whose levels increase in response to stress in order to trigger proapoptotic pathways. ${ }^{8,9}$ The activation of autophagy, often in response to environmental stresses such as starvation for key nutrients, appears to be another common mechanism involved in promoting the survival of cells in a number of stressful circumstances. ${ }^{10-12}$ In addition to its cytoprotective role, autophagy is also well known as type II PCD. ${ }^{13}$ There remains a great deal of controversy regarding how widespread autophagic cell death is with increasing consensus that autophagic cell death may be a form of apoptotic or type I PCD. ${ }^{14-16}$ Alternatively, autophagic PCD may occur at the end of prolonged or acute periods of autophagy. ${ }^{17}$ Yeast has proven useful for delineating the basic framework of autophagy and this combined with its emergence as genetic model for PCD suggest that yeast will prove to be a useful tool to understand autophagic death. ${ }^{7,18-21}$ Here we report that a previously identified Bax suppressor $14-3-3 \beta / \alpha$ is a functionally novel antiapoptotic sequence. ${ }^{22}$ We use 14-3-3 $\beta / \alpha$ along with yeast mutants defective in macroautophagy as tools to explore cross-talk between autophagic cell death and antiapoptosis.

\section{Results}

Identification of human 14-3-3 $\beta / \alpha$ as a Bax suppressor. We report the characterization of a Bax suppressor identified in a previous screen, a $0.999 \mathrm{~Kb}$ cDNA called Bh113. ${ }^{22}$ To confirm the results of the screen, wild-type yeast were re-transformed with the vector expressing Bax with empty vector, with Bh113 or the previously characterized dUTPase. $^{8}$ The three transformants were serially diluted and spotted on selective nutrient agar with glucose or galactose. As expected, cells harboring the Bax-containing plasmid

\footnotetext{
${ }^{1}$ Department of Chemistry and Chemical Engineering, Royal Military College, Kingston, Ontario, Canada; ${ }^{2}$ Institute for Molecular Biosciences, University of Graz, Graz, Austria and ${ }^{3}$ Department of Anatomy and Cell Biology, McGill University, Montreal, Quebec, Canada

${ }^{*}$ Corresponding author: MT Greenwood, Department of Chemistry and Chemical Engineering, Royal Military College, PO Box 17000, Station Forces, Kingston, Ontario K7K 7B4, Canada. Tel: +16136416000 Ext. 3575; Fax: + 1613542 9489; E-mail: michael.greenwood@ rmc.ca

${ }^{4}$ These authors contributed equally to this work.

Keywords: autophagy; antiautophagic; Bmh1; rapamycin; antiapoptotic; cell survival

Abbreviations: DHE, dihydroethidium; dUTP, deoxyuridne triphosphate; MEF, mouse embryonic fibroblast; PCD, programmed cell death; PI, propidium iodide; ROS, reactive oxygen species; TOR, target of rapamycin

Received 16.5.12; revised 11.6.12; accepted 13.6.12; Edited by G Raschellà
} 
failed to grow on the galactose inductive media. In contrast, the cells harboring the Bax along with Bh113- or dUTPaseexpressing plasmids showed significant growth on galactose (Supplementary Figure S1).

Analysis of the Bh113 nucleotide sequence revealed that it is an exact match to a portion of the 3015 nucleotide cDNA sequence encoding human 14-3-3 $\beta / \alpha$ (GenBank accession no. NM_139323). The coding sequence of the protein within the 14-3-3 $\beta / \alpha$ NM_139323 cDNA is located between nucleotides 188 and 928 . Our Bh113 sequence encompasses nucleotides $15-1014$ of the $14-3-3 \beta / \alpha$ sequence. Thus, our Bh133 sequence contains the entire coding sequence of the 246 amino acids of the $14-3-3 \beta / \alpha$ protein. The $14-3-3 \beta / \alpha$ protein is part of a family of proteins that have numerous chaperone-like functions, including the ability to prevent cell death. ${ }^{23}$ As previously demonstrated for a number of other sequences, the identification of $14-3-3 \beta / \alpha$ as a Bax suppressor in yeast suggests that it may be a powerful antiapoptotic protein. ${ }^{24}$

Human 14-3-3 $\beta / \alpha$ prevents cadmium-mediated cell death in yeast. To investigate the potential of $14-3-3 \beta / \alpha$ in yeast, we used the fact that cadmium can induce a PCD that is reversed by the expression of a previously characterized Bax suppressor. $^{8}$ Cultures of yeast transformed with different plasmids were diluted and aliquots spotted on nutrient agar galactose-containing plates with or without cadmium. Yeast cells harboring the $14-3-3 / / \alpha$ or the dUTPase cDNAs are more resistant to the inhibitory effects of cadmium as compared with control cells harboring the empty vector (Figure 1a). To ascertain if $14-3-3 \beta / \alpha$ actually prevents cadmium-mediated death, we grew cells in liquid media in the presence of cadmium and viability was determined using a vital dye. Cadmium decreased viability to $39.4 \pm 2.3 \%$ in controls, while 14-3-3 $\beta / \alpha$-expressing cells were protected with $79.1 \pm 2.1 \%$ viability (Figure $1 b$ ).

$14-3-3 \beta / \alpha$ is antiapoptotic. As a first step in characterizing the types of cell death that could be regulated by $14-3-3 \beta / \alpha$, we used cycloheximide a known inducer of apoptosis. ${ }^{25}$ Yeast cultures were treated with cycloheximide and their viability was determined by examining the proportion of cells that formed colonies (Figure 1c). Cycloheximide was dose dependent with a 36 and $11 \%$ remaining viable with 1 and $3 \mathrm{mM}$, respectively, in control cells. The viability was significantly increased to a respective 56 and $47 \%$ in cells expressing 14-3-3 $\beta / \alpha$ (Figure 1c). The accumulation of ROS is a typical feature of stressed cells and is also a typical hallmark of apoptosis. ${ }^{26}$ To monitor ROS we treated cells with dihydroethidium (DHE), a non-fluorescent compound that enters cells and becomes cleaved by ROS to generate fluorescent ethidium. We could thus demonstrate that cycloheximide-mediated increases in ROS levels are significantly reduced in cells expressing 14-3-3 $\beta / \alpha$ compared with cells harboring vector alone (Figure 1d). To further characterize 14-3-3 $\beta / \alpha$, we examined cells that were simultaneously stained with the nuclear vital dye propidium iodide (PI) and fluorescently labeled AnnexinV. Thus, we can detect cells that are undergoing cell surface exposure of phosphatidylserine (apoptosis; AnnexinV ${ }^{+}$) and loss of membrane integrity (necrotic cell death; $\left.\mathrm{PI}^{+}\right) .{ }^{27}$ In combination, this allows the discrimination between cells that are in early apoptosis (AnnexinV ${ }^{+} / \mathrm{PI}^{-}$), late apoptosis/secondary necrosis (AnnexinV ${ }^{+} / \mathrm{PI}^{+}$) and necrosis (AnnexinV $\mathrm{PII}^{+}$). Our analysis indicated that cycloheximide increased apoptotic markers which were significantly decreased in cells expressing 14-3-3 $\beta / \alpha$ (Figure 1e). Taken together, these results indicate that $14-3-3 \beta / \alpha$ is capable of preventing cell death that has the hallmarks of apoptosis.

14-3-3 $\beta / \alpha$ prevents rapamycin-mediated growth inhibition and cell death. To evaluate the possibility that 14-3-3 $\beta /$ $\alpha$ could serve to inhibit autophagic or type II PCD, we used conditions in which autophagy is activated in yeast. We initially used rapamycin, a common inhibitor of target of rapamycin (TOR), that inhibits cellular growth and activates autophagy. ${ }^{28}$ In yeast, the overexpression of 14-3-3 allows the cells to grow in the presence of rapamycin. ${ }^{29}$ Although its function is not known, the rapamycin-resistant phenotype is interpreted as 14-3-3 having a role in regulating TOR. ${ }^{30}$ Although rapamycin is routinely used to induce autophagy, it does have additional effects including inducing cell death. The type of cell death induced by rapamycin is widely seen as apoptotic-like, but the possibility that prolonged activation of autophagy by rapamycin may lead to an autophagic-like cell death that is still being intensively investigated. ${ }^{28,31}$ Thus, we sought to determine whether rapamycin-mediated death is a form of autophagic PCD and whether it is inhibited by overexpressing $14-3-3 \beta / \alpha$. We thus set out to determine the effects of expressing 14-3-3 $\beta / \alpha$ on the sensitivity of yeast cells to rapamycin. As a prelude we determined the minimum concentration of rapamycin needed to inhibit the growth of cells. Using the spot growth assay we demonstrate that $100 \mathrm{nM}$ of rapamycin induced a noticeable inhibition of growth in cells harboring the empty vector but that cells having the 14-3-3 $\beta / \alpha$-expressing plasmid grew normally (Supplementary Figure S2). Given that $14-3-3 \beta / \alpha$ can prevent cell death, this suggests that rapamycin induces growth arrest and cell death in yeast as it does in mammalian cells, and that $100 \mathrm{nM}$ is the threshold concentration that initiates death.

To directly evaluate the deadly impact of rapamycin treatment, we examined its effect on the viability of cells harboring empty vector or the plasmid expressing 14-3-3 $\beta / \alpha$. Rapamycin was added to growing cultures and aliquots of cells were removed daily and viability determined by examination after staining with vital dye. Although rapamycin decreased the viability of both cultures, cells expressing 14-3$3 \beta / \alpha$ maintained higher viability compared with control cells (Figure 1f). For example, viability decreased to $29.5 \pm 1.1 \%$ after 6 days of rapamycin treatment in cells harboring the empty vector, while the viability was $88.1 \pm 0.9 \%$ in $14-3-3 \beta / \alpha-$ expressing cells (Figure 1f). In the absence of rapamycin, viability of cells harboring empty vector remained high with $85 \pm 1.5 \%$ viability after 6 days. This reinforces the concept that rapamycin induces a death that is prevented by $14-3-3 \beta / \alpha$.

Autophagy is not required for $14-3-3 \beta / \alpha$ to protect against rapamycin. To investigate the role of autophagy 
a
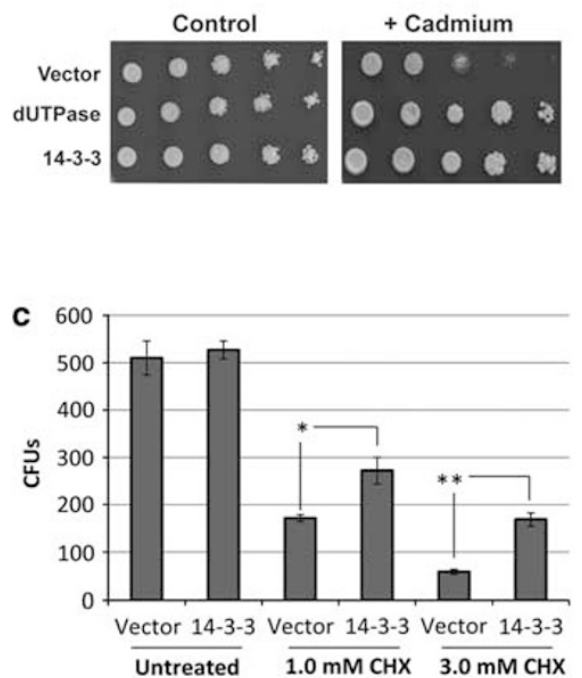

e

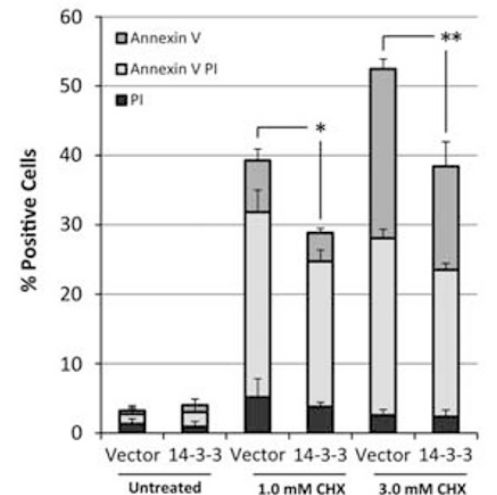

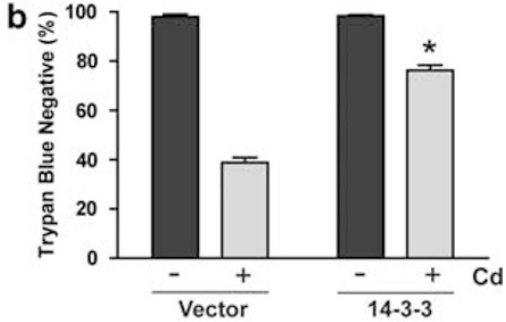

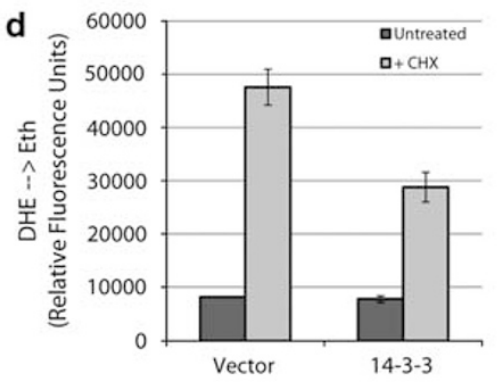

f

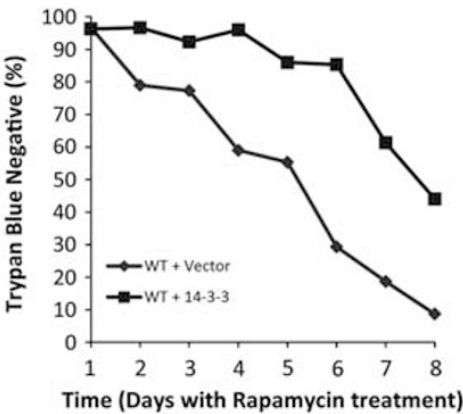

Figure $1 \quad 14-3-3 \beta$ protects against various triggers of cell death in yeast. (a) Freshly saturated cultures of yeast cells harboring control empty plasmid (Vector), as well as plasmids expressing the Bax suppressors dUTPase and 14-3-3/ $\beta$ (14-3-3) were serially diluted and aliquots were spotted onto YNB galactose-containing nutrient agar with no additions (Control) or with $1.5 \mathrm{mM}$ cadmium sulfate (+ Cadmium). The plates were incubated at $30^{\circ} \mathrm{C}$ for 3 days. (b) Freshly saturated cultures of yeast cells harboring control empty plasmid (Vector) and the plasmid expressing and 14-3-3 $/ / \alpha$ (14-3-3) were diluted and grown in galactose nutrient media for $4 \mathrm{~h}$. Cells were then grown for $6 \mathrm{~h}$ without (black bars) or with $1.5 \mathrm{mM}$ cadmium sulfate (gray bars). Viability was determined by microscopic examination of cells stained with the vital dye trypan blue. The data are shown as the percentage (\%) of cells that does not stain with trypan blue and are thus viable (trypan blue negative). The data represent the mean of four independent experiments ( \pm S.E.M.). ${ }^{*}$, indicates a significant difference between the viabilities of cadmium-treated Vector cells versus cadmium-treated 14-3-3-expressing cells (Student's $t$-test $P<0.001$ ). (c-e) Human 14-3-3 $/ \alpha$ prevents cycloheximide-mediated apoptosis. Freshly saturated cultures of yeast cells with empty plasmid (Vector) and the 14-3-3 $\beta /$ $\alpha$-expressing plasmid (14-3-3) were diluted and grown in galactose nutrient media for $4 \mathrm{~h}$ prior to the addition of cycloheximide. (c) Viability of the cells was determined after a $24 \mathrm{~h}$ treatment with the indicated concentrations of cycloheximide using the clonogenicity assay. The data represent the mean of four independent experiments $( \pm$ S.E.M.). ${ }^{*}$ and ${ }^{* *}$ indicates significant difference between the viabilities of control vector cells versus $14-3-3$-expressing cells $\left({ }^{\star} P<0.05\right.$ and $\left.{ }^{* *} P<0.01\right)$. (d) Fluorescence was monitored in DHE-treated cells using a fluorescent cell sorter. The results are reported as the mean ( \pm S.D.) of two experiments that were performed in triplicate. (e) Cells were simultaneously challenged with fluorescently labeled annexin $V$ and propidium iodide, and the proportion of cells labeled with either or with both were determined by cell sorter as described. ${ }^{27}$ The data represent the mean of four independent experiments ( \pm S.E.M.). ${ }^{*}$ and ${ }^{* *}$ indicates significant differences between the control vector cells versus 14-3-3-expressing cells $\left({ }^{*} P<0.05\right.$ and $\left.{ }^{* *} P<0.01\right)$. (f) Rapamycin-mediated cell death is inhibited by human $14-3-3 \beta / \alpha$. Freshly saturated cultures of yeast cells with empty plasmid (circles) and the 14-3-3 $\beta / \alpha$-expressing plasmid (squares) were diluted and grown in galactose nutrient media for $4 \mathrm{~h}$. Rapamycin was added (500 $\mathrm{nM}$ ) and viability was monitored daily using the vital dye trypan blue over an 8-day period. The data are shown as the percentage (\%) of cells that does not stain with trypan blue (trypan blue negative). The results are reported as the mean ( \pm S.D.) of two experiments that were performed in triplicate

in rapamycin resistance, we used mutants lacking the genes encoding Atg1p and Atg7p, as they are able to grow normally but are unable to carry out macroautophagy, the form of autophagy that is induced by rapamycin and the form that offers the most striking protection against stress. ${ }^{18}$ In effect, these strains show an increased lethality in the face of numerous stresse,s including starvation for leucine (see below, Figures 2c and d). We also used a atg114, as it grows normally, has normal macroautophagy and instead it is selectively defective in the minor autophagic pathway for mitophagy. ${ }^{18}$ The atg 4 and wild-type strains with empty vector and the 14-3-3 $\beta / \alpha$-expressing plasmid show similar growth in the absence of the drug, whereas all the strains expressing 14-3-3 $\beta / \alpha$ show enhanced growth in the presence of rapamycin when compared with control cells (Figure 2a). These results suggest that enhanced activation of autophagy 

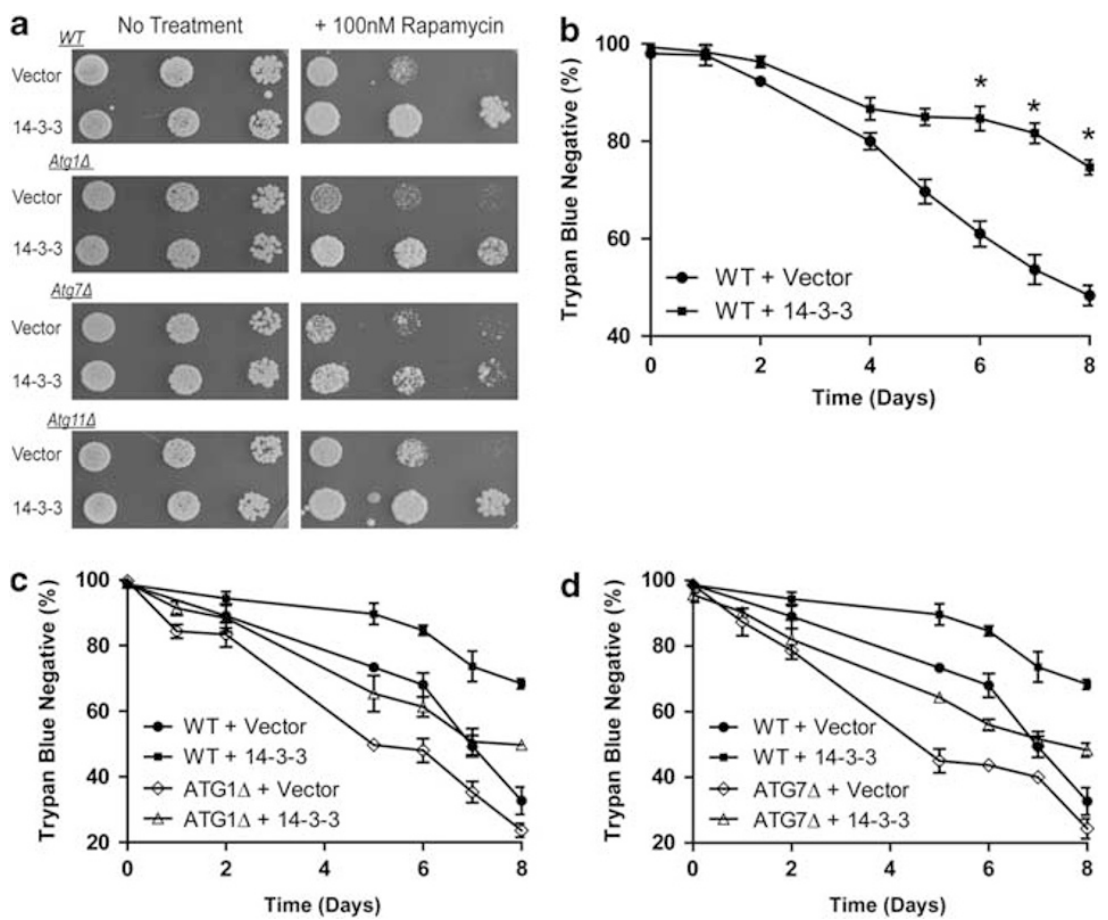

Figure 2 Cell death due to prolonged autophagy is rescued by 14-3-3. (a) 14-3-3-mediated rapamycin resistance is autophagy independent. Freshly saturated cultures of different yeast strains including wild type (WT) as well as the isogenic derivatives that are lacking the Atg1, Atg7 and Atg11 genes were individually transformed with control empty plasmid (Vector), and the plasmid expressing 14-3-3 $\beta / \alpha$ (14-3-3) were serially diluted and aliquots were spotted onto YNB galactose-containing nutrient agar with no additions (no treatment) or with $100 \mathrm{nM}$ rapamycin. The plates were incubated at $30^{\circ} \mathrm{C}$ for 2 days (no treatment) or for 4 days ( $+100 \mathrm{nM}$ rapamycin). (b-d) $14-3-3$ confers resistance to leucine starvation-mediated cell death in an autophagy-independent manner. Freshly saturated cultures of the leucine auxotrophic wild-type yeast cells harboring different plasmids were diluted and grown in complete minimal galactose-containing nutrient media for $4 \mathrm{~h}$, washed with sterile water and resuspended at $2 \times 10^{6} \mathrm{cells} / \mathrm{ml}$ in complete minimal galactose-containing media without leucine. The cultures were incubated at $30^{\circ} \mathrm{C}$ and samples were taken at intervals to determine viability using the vital dye trypan blue. The data are shown as the percentage (\%) of cells that does not stain with trypan blue (trypan blue negative). (b) Wild-type yeast cells with control empty plasmid (full circles) as well as the plasmid expressing 14-3-3 $\beta / \alpha$ (full squares) underwent leucine starvation and viability was determined. The data represent the mean of four independent experiments ( \pm S.E.M.). *indicates a significant difference between the viabilities of control vector cells versus 14-3-3-expressing cells (Student's t-test $P<0.001$ ). (c and d) Wild type as well as mutants that are lacking the atg1 (open diamonds) and atg7 (open triangles) genes were individually transformed with the empty vector and the plasmid expressing 14-3-3/ $\alpha$ (14-3-3), and viability was determined following leucine deprivation. The data are shown as the percentage (\%) of cells that does not stain with trypan blue (trypan blue negative). The experiments presented in $\mathbf{c}$ and $\mathbf{d}$ are the mean $( \pm S$.D.) of two experiments that were performed in triplicate. The data are presented in two separate panels for presentation purposes. The data for the WT cells (WT + Vector; WT $+14-3-3)$ are thus the same for $\mathbf{c}$ and $\mathbf{d}$, but the data presented in $\mathbf{b}$ for these same strains were obtained from independent experiments

does not account for the protective effects of $14-3-3 \beta / \alpha$ and that the activation of autophagy is not required to mediate the inhibitory effects of rapamycin (Figure 2a).

\section{Human 14-3-3 $\beta / \alpha$ prevents leucine starvation-mediated} cell death. We used leucine deprivation as an alternate method to activate autophagy and allow cell death to occur when the cells are 'exhausted' or depleted due to chronic autophagy. ${ }^{18,32,33}$ Thus, cultures of wild-type strain with empty vector or plasmid expressing $14-3-3 \beta / \alpha$ were subjected to leucine deprivation and viability was determined using vital dye. Viability of control cells decreased gradually over time and this was significantly blunted in cells expressing 14-3-3 $\beta / \alpha$ (Figure $2 b$ ). In effect, by day 6 , viability is reduced to $61 \pm 2.6 \%$ in controls but is elevated to $84.7 \pm 2.5 \%$ in cells expressing $14-3-3 \beta / \alpha$ (Figure $2 b$ ). The observed decrease in viability is specifically due to the absence of leucine, as the viability of cells harboring empty vector was largely unaffected when grown in the presence of media with leucine with $86 \pm 2.1 \%$ remaining viable after 6 days. The autophagic-deficient atg1 14 and atg7 4 mutants without and with the 14-3-3 $\beta / \alpha$-expressing plasmid were also subjected to leucine deprivation. As expected, loss of viability was more pronounced in the autophagic mutants when compared with wild-type cells (Figures $2 c$ and $d$ ). At day 6 , the viability of wild type was decreased to $68 \pm 3.6 \%$, whereas the viability of the atg1 $\triangle 1$ and atg7 4 mutants was $48 \pm 3.6$ and $43.7 \pm 1.2 \% \%$, respectively (Figures $2 c$ and $d$ ). This shows that viability during leucine starvation is enhanced by a functional autophagic pathway. The viability was enhanced in the wild-type and autophagic mutant strains by the expression of $14-3-3 \beta / \alpha$ (Figures $2 c$ and $d$ ). This suggests that the observed cell death is not dependent on autophagy and that it is likely an apoptotic-type PCD.

Human $14-3-3 \beta / \alpha$ is physiologically relevant in yeast. Yeast cells have two distinct but almost identical 14-3-3 genes, $B M H 1$ and $B M H 2$, both of which appear to function as multifaceted chaperonin-like proteins much as the mammalian 14-3-3 genes (Supplementary Figure S3a). ${ }^{23,34}$ Human $14-3-3 / / \alpha$ and yeast $B m h 1 p$ share $63 \%$ sequence identity, which is higher than the $\sim 30 \%$ identity shared between most 
other yeast-human orthologs (Supplementary Figure S3b). ${ }^{35}$ Nevertheless, human $14-3-3 \beta / \alpha$ is still a heterologous gene and it may not be physiologically relevant in yeast. To examine if $14-3-3 \beta / \alpha$ is a functional 14-3-3 sequence, we examined if it could complement the rapamycin sensitivity of mutants lacking bmh1 or bmh2. ${ }^{29}$ Using the spot growth assay, we show that yeast cells lacking $B m h 1$ or $B m h 2$ show very little growth when compared with wild-type cells in the presence of rapamycin (Figure 3a). In contrast, Bmh1s, and to a lesser extent $B m h 2 \Delta$ cells, show enhanced growth when overexpressing 14-3-3 $\beta / \alpha$. These results suggest that $14-3-3 \beta / \alpha$ is a functional ortholog of the yeast $14-3-3$ genes.

The yeast ortholog of human 14-3-3, BMH1, is antiapoptotic. Antiapoptotic genes are characterized by the increased and decreased resistance to apoptotic stresses, respectively, upon overexpression and knockout. ${ }^{5}$ To determine whether the yeast $B M H$ genes are also antiapoptotic, we first examined the effects of apoptosis inducing levels of cycloheximide on yeast cells lacking Bmh14 and Bmh24 genes. Yeast cells lacking the $B M H 1$ gene show all the signs of being hypersensitive to the stress of cycloheximide (Figures $3 b-d$ ). When challenged with apoptotic-inducing stress they show an enhanced increase in their levels of ROS (Figure 3b), they show a decrease in their viability (Figure 3c) and they show increase in the percentage of cells that have overt hallmarks of apoptosis including staining with annexin $\mathrm{V}$ (Figure $3 \mathrm{~d}$ ). In contrast, Bmh24 cells are not hypersensitive to the apoptotic-inducing stress as we observed very little differences when compared with wild-type cells a

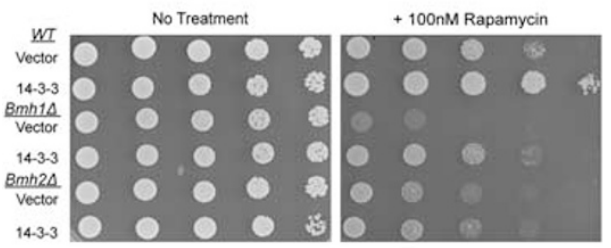

b
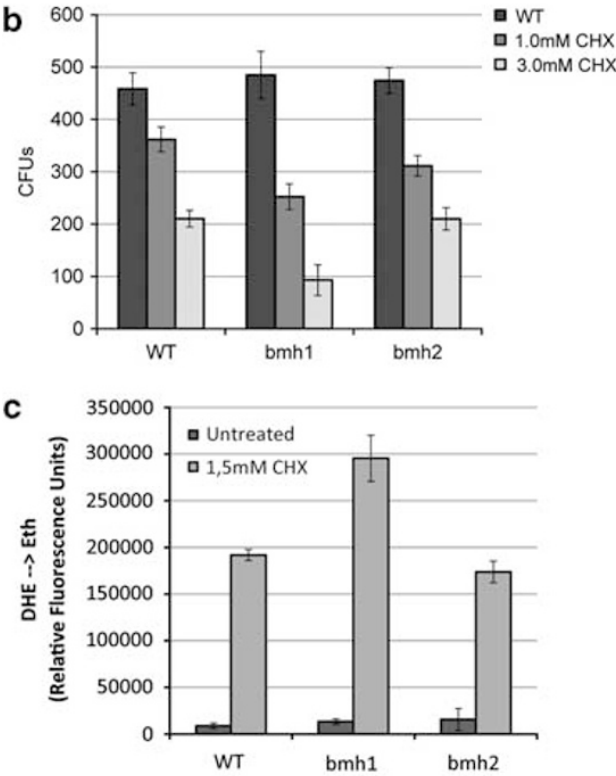

d
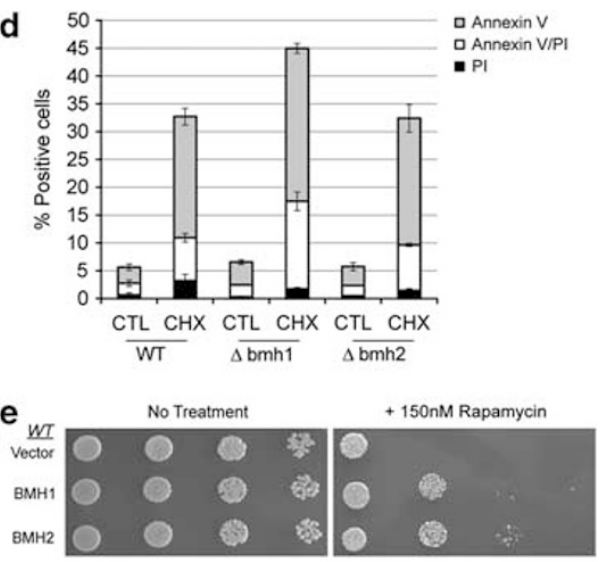

Bmh1s
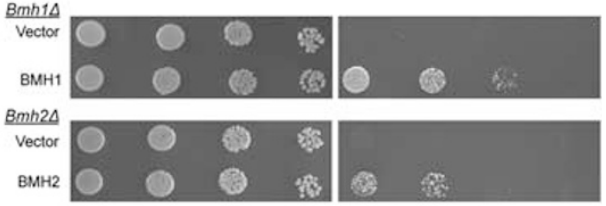

f

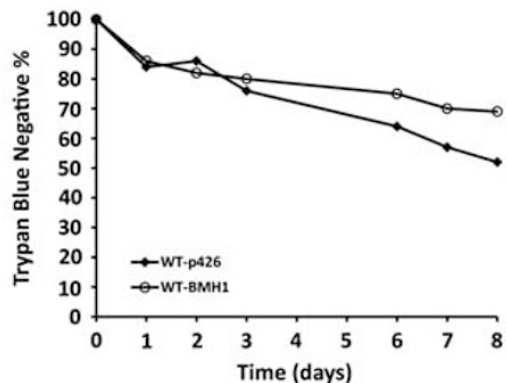

Figure 3 Yeast and human 14-3-3 proteins are functionally interchangeable. (a) Human 14-3-3 $\beta / \alpha$ can functionally replace the yeast $B M H 1$ and $B M H 2$ encoding the 14-33 genes. Freshly saturated cultures of different yeast strains including wild type (WT) as well as the isogenic derivatives that are lacking the yeast 14-3-3 Bmh1 or Bmh2 (Bmh14 or Bmh24) genes transformed with different plasmids (Vector, 14-3-3) were serially diluted and aliquots were spotted onto galactose-containing nutrient agar without (no treatment) or with $100 \mathrm{nM}$ rapamycin and the plates were incubated at $30^{\circ} \mathrm{C}$. (b-d) Yeast mutants lacking the $B M H 1$ gene are hypersensitive to apoptotic-inducing stress. Yeast mutants lacking the Bmh1 $\triangle$ and Bmh2 $\triangle$ genes were treated with cycloheximide and different apoptotic parameters determined. The results are reported as the mean ( \pm S.D.) of two experiments that were performed in triplicate. (b) Viability of the cells was determined after $5 \mathrm{~h}$ with cycloheximide using the clonegicity assay. (c) Fluorescence was monitored in DHE-treated cells using a fluorescent cell sorter. (d) Cells were simultaneously challenged with fluorescently labeled annexin $V$ and propidium iodide, and the proportion of cells labeled with either or with both were determined by cell sorter as described. ${ }^{27}$ (e) Rapamycin-mediated cell death is inhibited by yeast 14-3-3. Spot assays were carried out using freshly saturated cultures of wild-type cells (WT) and mutants lacking 14-3-3 (Bmh14 and Bmh2A) transformed with control plasmid (Vector) or plasmids overexpressing the yeast 14-3-3 genes (BMH1 and BMH2). The cells were spotted onto galactose-inducible selectable media without (no treatment) or with $150 \mathrm{nM}$ rapamycin ( + Rapamycin). ${ }^{*} P<0.05$ and ${ }^{* *} P<0.01$. (f) Viability was determined, using the vital dye trypan blue, following leucine deprivation in wild-type cells transformed with the empty vector (open diamonds) and the plasmid expressing yeast BMH1 (open triangles). The data are shown as the percentage (\%) of cells that does not stain with trypan blue (trypan blue negative). The results are reported as the average of two experiments that were performed in triplicate 
(Figures $3 b-d)$. These results are consistent with previous observations that the $B M H 1$ gene is likely the more overtly important of the two yeast 14-3-3 genes. ${ }^{34}$

We then used yeast cells overexpressing both $B M H$ genes to determine whether the sequences could also serve to protect against apoptotic-inducing stress. Using the spot assay, it is clear that wild-type yeast overexpressing either $B M H 1$ or $B M H 2$ show an increase in the resistance to the growth inhibitory effects of rapamycin (Figure $3 e$ ). Thus, both genes appear to have antiapoptotic functions when overexpressed. Yeast cells overexpressing BMH1 also show increased viability compared with control cells in response to prolonged periods of autophagy induced by leucine starvation (Figure $3 f$ ). The viability of control cells was decreased to $52 \pm 2.3 \%$ compared with $69 \pm 3.5 \%$ after 8 days of leucine deprivation. Taken together, the results in Figure 3 indicate that at least one of the yeast 14-3-3 proteins, Bmh1p, is an antiapoptotic gene that has similar properties to human $14-3-3 \beta / \alpha$.

\section{Discussion}

The process by which cells protect themselves from inadvertently and prematurely inducing cell death in response to sublethal stresses involves a coordinated response to prevent the initiation of cell death. ${ }^{1,5}$ One common method is the use of antiapoptotic proteins that inhibit well-characterized proapoptotic pathways. Thus, Bcl-2 is a potent inhibitor of Bax such that the level of functional $\mathrm{Bcl}-2$ serves as a threshold for Bax-mediated cell death (Figure 4). This is evident from the fact that decreases or increases in the levels of $\mathrm{Bcl}-2$ changes threshold for stress and their ability to prevent apoptotic cell death. ${ }^{36}$ Here we show that human $14-3-3 \beta / \alpha$ is capable of preventing cell death that has the hallmarks of apoptosis in yeast (Figures 1 and 2). Although the mechanisms are not yet known, $14-3-3 \beta / \alpha$ is a chaperonin-type protein that likely alleviates cellular stress and subsequent activation of apoptotic pathways by assisting damaged proteins to maintain their physiologically relevant structure. Other chaperonintype proteins that are antiapoptotic include a variety of heatshock proteins, and some of these proteins may promote cell survival by binding to and inhibiting proapoptotic proteins. ${ }^{37}$ The availability of yeast-based antiapoptosis assay system to study human $14-3-3 \beta / \alpha$ will facilitate a genetic determination of the structural motifs and the cofactors required for its prosurvival function.

The processes by which autophagic-mediated cell death occurs has remained elusive and many of the experimental evidence used for its detection are in fact quite controversial. ${ }^{14-16}$ It is of interest that many studies have shown that negative regulators of autophagic death do not delay cell death at the end of autophagy, but instead they prevent the induction of autophagy. There are very few studies that actually show a causal relationship between cell death and autophagy. ${ }^{5,13}$ We thus examined the use of the 14-3-3 antiapoptotic sequence to determine whether it could prevent cell death under culture conditions where autophagy is known to be actually occurring. We thus could demonstrate that wellknown conditions that serve to activate autophagy, the specific inhibition of TOR 1 using rapamycin and starvation for the amino acid leucine both lead to cell death which could be inhibited by the expression of human 14-3-3 $\beta / \alpha$ (Figure 2).

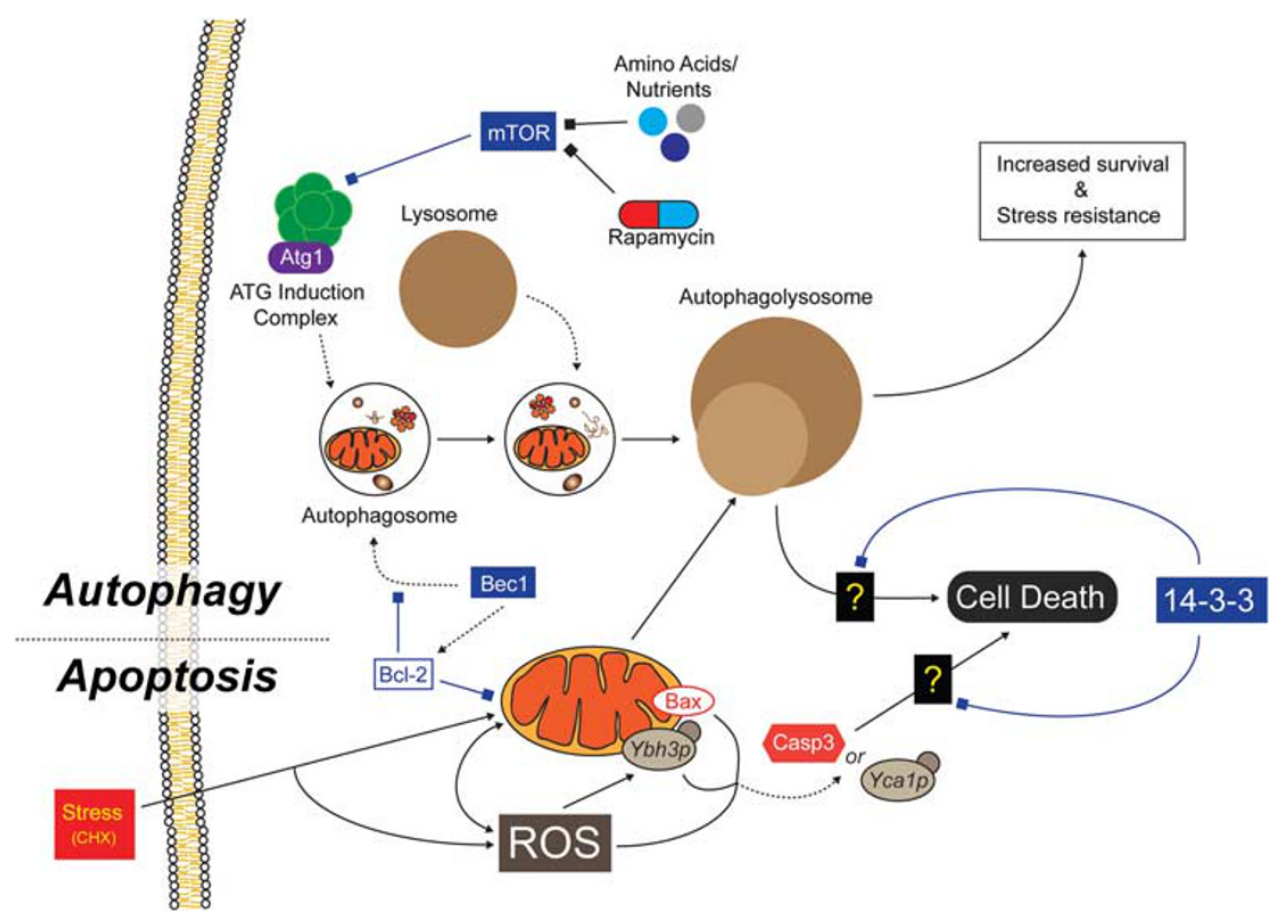

Figure 4 Schematic representation of proteins that regulate apoptosis and autophagy. The typical autophagic pathway including the role of $m$ TOR and its inactivation by nutrients and rapamycin is shown on top. The role of the Atg1p in initiating autophagy is depicted. Proteins and effectors including ROS, Bcl-2 and Bec1 (Beclin1) that are involved in both apoptosis and autophagy are shown. This study shows that 14-3-3 can inhibit cell death due to stimuli that induce apoptosis and by stimuli that are commonly used to induce autophagy 
Cells lacking the autophagic genes atg 1 or atg 7 were still able to undergo cell death with rapamycin or if starved for leucine and that this death could be still be prevented by the expression of human 14-3-3 $\beta / \alpha$ (Figure 2). This supports the notion that the cell death may not be due to autophagy. It does nevertheless remain possible that blocking autophagy may switch the cell from autophagic to apoptotic cell death and that $14-3-3 \beta / \alpha$ is able to serve to inhibit both type I and II PCD (Figure 4). This reflects the fact that there is a great deal of cross-talk between apoptosis and autophagy with the most well-known cases involving the ability of activated autophagy to inhibit apoptosis. ${ }^{5,14}$ Other interesting examples involve apoptotic-resistant cells, such as mouse embryonic fibroblasts (MEFs) that are derived from embryos having a double knockout of the two genes encoding the proapoptotic $\mathrm{Bcl}-2$ proteins Bax and Bak. These cells are reported to undergo autophagic cell death in response to apoptotic stimuli. ${ }^{38}$ In spite of this we nevertheless favor an increasingly accepted scenario that autophagic cell death per se is a rare event, instead what is commonly observed is really a form of apoptosis that is associated with autophagy or possibly triggered by autophagy. ${ }^{14,16}$ The intense ongoing investigations into the processes of autophagy will lead to novel assays and markers that will eventually serve to resolve the confusion regarding the importance of autophagic death. $2,14,16$ The inability to find a true inducer of autophagic cell death in a screen that identified $\sim 1400$ chemical inducers of PCD serves as strong support. ${ }^{39}$ An alternative approach would be to identify specific inhibitors of autophagic cell death, possibly by screening cDNA or chemical libraries in cells undergoing PCD after prolonged periods of autophagy. Identification of such specific prosurvival chemicals and proteins for cells undergoing necrosis provided a strong rational for the support of genetically programmed forms of necrosis. ${ }^{19,27}$

\section{Materials and Methods}

Yeast strains and plasmids. The $S$. cerevisiae BY4742 (MATa his $3 \Delta 1$ leu2 $\Delta 0$ lys $2 \Delta 0$ ura3 $\Delta 0$ ) was used as the wild-type strain, and the mutant strains used were isogenic except for the deletion of an autophagic gene (atg1 14 , atg7 $\triangle$ and atg114) or of the yeast 14-3-3 encoding genes (bmh14 and bmh2A) (EUROSCARF). The 14-3-3 $\beta / \alpha$ and dUTPase encoding Bax suppressors, expressed under the control of the galactose-inducible GAL1 promoter in pYESDEST52, were isolated in our previous Bax suppressor screen of a human cardiac CDNA library. ${ }^{8,22}$ The galactose-inducible $B M H 1$ and $B M H 2$ overexpressing plasmids were obtained from Open Biosystems (Thermo Scientific, Lafayette, CO, USA).

Yeast transformations, growth, viability and cell death assays. Yeast were grown in synthetic minimal media containing yeast nitrogen base (YNB), $2 \%$ glucose and supplemented with the required amino acids or bases. When expression of the GAL1 promoter was required, the glucose was substituted with $2 \%$ galactose and $2 \%$ raffinose. Plasmids were introduced into yeast cells using lithium acetate and transformants were selected for by removing the appropriate nutrient. Two methodologies were used to determine viability. The clonogenicity assay consisted of removing aliquots of differentially treated cells serially diluted and triplicate samples of 200-500 cells were then plated on YEPD media, grown at $30^{\circ} \mathrm{C}$ and the number of colonies that formed after 2 days were counted. ${ }^{22,27}$ Viability was also determined by microscopical examination of cells treated with the vital dye trypan blue. ${ }^{22}$ Cells were placed in a final concentration of $0.1 \%$ trypan blue for $5 \mathrm{~min}$ and at least 300 cells were scored for each time point. Treatment of cells in liquid cultures consisted of generating freshly saturated overnight cultures of the different yeast transformants, which were then diluted in fresh, pre-warmed galactose-containing media, incubated for $4 \mathrm{~h}$ to induce GAL1 gene expression, and subsequently treated with the indicated concentrations of compound including cadmium, cycloheximide and rapamycin. ${ }^{22}$ For the spot growth assays, freshly saturated cultures grown in glucose media, serially diluted 5 -fold and $5 \mu \mathrm{l}$ of the appropriate dilutions were spotted onto nutrient-containing galactose with and without the different chemical stresses. All spot assays were performed a minimum of three times with identical results. Flow cytometry using 30,000 cells per sample were used for cell death marker assays, including DHE staining (ROS production) and Annexin V/PI containing (apoptosis/necrosis marker) as previously described. ${ }^{27}$

\section{Conflict of Interest}

The authors declare no conflict of interest.

Acknowledgements. This work was supported by a grant from NSERC to MTG, and the Heart and Stroke Foundation of Canada to CAM and MTG. FM is grateful to the FWF for grants LIPOTOX, DK-MCD W 1226- B18, P23490-B12 and P24381-B20.

1. Fulda S, Gorman AM, Hori O, Samali A. Cellular stress responses: cell survival and cell death. Int J Cell Biol 2010; Article ID 214074.

2. Galluzzi L, Vitale I, Abrams JM, Alnemri ES, Baehrecke EH, Blagosklonny MV et al. Molecular definitions of cell death subroutines: recommendations of the Nomenclature Committee on Cell Death 2012. Cell Death Differ 2012; 19: 107-120.

3. Strasser A, Cory S, Adams JM. Deciphering the rules of programmed cell death to improve therapy of cancer and other diseases. EMBO J 2011; 30: 3667-3683.

4. Lavu M, Gundewar S, Lefer DJ. Gene therapy for ischemic heart disease. J Mol Cell Cardiol 2011; 50: 742-750

5. Portt L, Norman G, Clapp C, Greenwood M, Greenwood MT. Anti-apoptosis and cell survival: a review. Biochim Biophys Acta 2011; 1813: 238-259.

6. Christofferson DE, Yuan J. Necroptosis as an alternative form of programmed cell death. Curr Opin Cell Biol 2010; 22: 263-268.

7. Eisenberg T, Carmona-Gutierrez D, Buttner S, Tavernarakis N, Madeo F. Necrosis in yeast. Apoptosis 2010; 15: 257-268.

8. Williams D, Norman G, Khoury C, Metcalfe N, Briard J, Laporte A et al. Evidence for a second messenger function of dUTP during Bax mediated apoptosis of yeast and mammalian cells. Biochim Biophys Acta 2011; 1813: 315-321.

9. Wilson PM, Labonte MJ, Lenz HJ, Mack PC, Ladner RD. Inhibition of dUTPase induces synthetic lethality with thymidylate synthase-targeted therapies in non-small cell lung cancer. Mol Cancer Ther 2012; 11: 616-628.

10. Marino G, Madeo F, Kroemer G. Autophagy for tissue homeostasis and neuroprotection. Curr Opin Cell Biol 2011; 23: 198-206.

11. Morselli E, Galluzzi L, Kepp O, Vicencio JM, Criollo A, Maiuri MC et al. Anti- and pro-tumor functions of autophagy. Biochim Biophys Acta 2009; 1793: 1524-1532.

12. Meschini S, Condello M, Lista P, Arancia G. Autophagy: molecular mechanisms and their implications for anticancer therapies. Curr Cancer Drug Targets 2011; 11: 357-379.

13. Sampaio-Marques B, Felgueiras C, Silva A, Rodrigues F, Ludovico P. Yeast chronological lifespan and proteotoxic stress: is autophagy good or bad? Biochem Soc Trans 2011; 39: 1466-1470.

14. Shen S, Kepp O, Kroemer G. The end of autophagic cell death? Autophagy 2012; 8: 1-3.

15. Levine B, Kroemer G. Autophagy in aging, disease and death: the true identity of a cell death impostor. Cell Death Differ 2009; 16: 1-2.

16. Denton D, Nicolson S, Kumar S. Cell death by autophagy: facts and apparent artefacts. Cell Death Differ 2012; 19: 87-95

17. Scarlatti F, Granata R, Meijer AJ, Codogno P. Does autophagy have a license to kill mammalian cells? Cell Death Differ 2009; 16: 12-20.

18. He C, Klionsky DJ. Regulation mechanisms and signaling pathways of autophagy. Annu Rev Genet 2009; 43: 67-93.

19. Carmona-Gutierrez D, Eisenberg T, Buttner S, Meisinger C, Kroemer G, Madeo F. Apoptosis in yeast: triggers, pathways, subroutines. Cell Death Differ 2010; 17: 763-773.

20. Zdralevic M, Guaragnella N, Antonacci L, Marra E, Giannattasio S. Yeast as a tool to study signaling pathways in mitochondrial stress response and cytoprotection. Scientific World J 2012; Article ID 912147.

21. Munoz AJ, Wanichthanarak K, Meza E, Petranovic D. Systems biology of yeast cell death. FEMS Yeast Res 2012; 12: 249-265.

22. Yang Z, Khoury C, Jean-Baptiste G, Greenwood MT. Identification of mouse sphingomyelin synthase 1 as a suppressor of Bax-mediated cell death in yeast. FEMS Yeast Res 2006; 6: 751-762.

23. Gardino AK, Yaffe MB. 14-3-3 proteins as signaling integration points for cell cycle control and apoptosis. Semin Cell Dev Biol 2011; 22: 688-695.

24. Greenwood MT, Ludovico P. Expressing and functional analysis of mammalian apoptotic regulators in yeast. Cell Death Differ 2010; 17: 737-745. 
25. Donovan MJ, Maciuba BZ, Mahan CE, McDowell MA. Leishmania infection inhibits cycloheximide-induced macrophage apoptosis in a strain-dependent manner. Exp Parasitol 2009; 123: 58-64.

26. Madeo F, Frohlich E, Ligr M, Grey M, Sigrist SJ, Wolf DH et al. Oxygen stress: a regulator of apoptosis in yeast. J Cell Biol 1999; 145: 757-767.

27. Buttner S, Ruli D, Vogtle FN, Galluzzi L, Moitzi B, Eisenberg T et al. A yeast BH3-only protein mediates the mitochondrial pathway of apoptosis. EMBO J 2011; 30: 2779-2792.

28. Galluzzi L, Morselli E, Kepp O, Vitale I, Younes AB, Maiuri MC et al. Evaluation of rapamycin-induced cell death. Methods Mol Biol 2012; 821: 125-169.

29. Bertram PG, Zeng C, Thorson J, Shaw AS, Zheng XF. The 14-3-3 proteins positively regulate rapamycin-sensitive signaling. Curr Biol 1998; 8: 1259-1267.

30. Lee JW, Park S, Takahashi Y, Wang HG. The association of AMPK with ULK1 regulates autophagy. PLOS One 2010; 5: e15394.

31. Yellen P, Saqcena M, Salloum D, Feng J, Preda A, Xu L et al. High-dose rapamycin induces apoptosis in human cancer cells by dissociating mTOR complex 1 and suppressing phosphorylation of 4E-BP1. Cell Cycle 2011; 10: 3948-3956.

32. Xu M, Zhang HL. Death and survival of neuronal and astrocytic cells in ischemic brain injury: a role of autophagy. Acta Pharmacol Sin 2011; 32: 1089-1099.

33. Yang Z, Huang J, Geng J, Nair U, Klionsky DJ. Atg22 recycles amino acids to link the degradative and recycling functions of autophagy. Mol Biol Cell 2006; 17: 5094-5104.

34. Wang C, Skinner C, Easlon E, Lin SJ. Deleting the 14-3-3 protein Bmh1 extends life span in Saccharomyces cerevisiae by increasing stress response. Genetics 2009; 183: 1373-1384.
35. Khoury CM, Yang Z, Ismail S, Greenwood MT. Characterization of a novel alternatively spliced human transcript encoding an N-terminally truncated Vps24 protein that suppresses the effects of Bax in an ESCRT independent manner in yeast. Gene 2007; 391: 233-241.

36. Henderson S, Rowe M, Gregory C, Croom-Carter D, Wang F, Longnecker R et al. Induction of bcl-2 expression by Epstein-Barr virus latent membrane protein 1 protects infected B cells from programmed cell death. Cell 1991; 65: 1107-1115.

37. Sreedhar AS, Csermely P. Heat shock proteins in the regulation of apoptosis: new strategies in tumor therapy: a comprehensive review. Pharmacol Ther 2004; 101: 227-257.

38. Shimizu S, Kanaseki T, Mizushima N, Mizuta T, Arakawa-Kobayashi S, Thompson CB et al. Role of Bcl-2 family proteins in a non apoptotic programmed cell death dependent on autophagy genes. Nat Cell Biol 2004; 6: 1221-1228.

39. Shen S, Kepp O, Michaud M, Martins I, Minoux H, Métivier D et al. Association and dissociation of autophagy, apoptosis and necrosis by systematic chemical study. Oncogene 2011; 30: 4544-4556.

Cell Death and Disease is an open-access journal published by Nature Publishing Group. This work is licensed under the Creative Commons Attribution-NonCommercial-No Derivative Works 3.0 Unported License. To view a copy of this license, visit http://creativecommons.org/licenses/by-nc-nd/3.0/

Supplementary Information accompanies the paper on Cell Death and Disease website (http://www.nature.com/cddis) 